\title{
Does Rheumatoid Arthritis Really Improve During Pregnancy? A Systematic Review and Metaanalysis
}

\author{
Hannah Jethwa, Suzanne Lam, Colette Smith, and Ian Giles
}

ABSTRACT. Objective. We performed a systematic review and metaanalysis to assess rheumatoid arthritis (RA)
disease activity during pregnancy using objective disease activity scoring systems.
Methods. A systematic review of PubMed, EMBASE/Medline, Cochrane, and LactMed databases
was performed. Our inclusion criteria for analysis were prospective studies, more than 5 patients per
study, and data on RA using an objective scoring system conducted by a clinician/health professional.
Results. Ten studies were eligible for final analysis, which included 237 patients, of which prepartum
data were available for 204 patients. Postpartum disease activity was recorded in 135 pregnancies.
Conclusion. Disease activity improved in $60 \%$ of patients with RA in pregnancy and flared in $46.7 \%$
postpartum. (First Release November $12018 ;$ J Rheumatol 2019;46:245-50; doi:10.3899/jrheum.180226)

Key Indexing Terms:

INFLAMMATORY ARTHRITIS

RHEUMATOID ARTHRITIS

PREGNANCY

DISEASE ACTIVITY PROSPECTIVE

The effects of pregnancy on inflammatory arthritis have been debated for many years, with early observations indicating a beneficial response in rheumatoid arthritis (RA) dating back to the 19th century ${ }^{1}$. In particular, many studies have reported that disease activity improves in up to $90 \%$ of patients with RA during pregnancy with a risk of subsequent postpartum flare $^{2}$.

Historical data, however, are based mostly on retrospective studies, which lack standardized and objective measures of disease activity. More recent prospective studies of patients with RA using objective disease activity scores demonstrate more modest improvements in disease activity during pregnancy.

RA often affects women of childbearing age, who frequently have concerns about the potential effect of

From the Rheumatology Department, London North West Healthcare National Health Service (NHS) Trust; General Medicine, Croydon University Hospital; Statistics Department, Royal Free Hospital; Centre for Rheumatology Research, Rayne Institute, University College London, London, UK.

This work was undertaken at University College London Hospitals, which received a proportion of funding from the UK Department of Health's National Institute for Health Research Biomedical Research Centres funding scheme.

H. Jethwa, Specialist Registrar in Rheumatology, BSc, MBChB, MRCP, Rheumatology Department, London North West Healthcare NHS Trust; S. Lam, General Practitioner Trainee, BSc, MBBS, General Medicine, Croydon University Hospital; C. Smith, Research Statistician, PhD, Statistics Department, Royal Free Hospital; I. Giles, Consultant Rheumatologist, PhD, FRCP, Centre for Rheumatology Research, Rayne Institute, University College London. H. Jethwa and S. Lam contributed equally to this study.

Address correspondence to H. Jethwa, Rheumatology Department,

Ealing Hospital, Uxbridge Road, Southall UB1 3HW, UK.

E-mail: hannahjethwa@nhs.net

Accepted for publication July 24, 2018. disease-modifying antirheumatic drugs (DMARD) upon their pregnancy. Accurate knowledge of the effect of pregnancy upon RA disease activity is therefore important to enable more-informed decision making during prepregnancy counseling to maintain DMARD that are compatible with pregnancy, to avoid disease relapse.

Therefore, we carried out this systematic review of prospective studies using serial, objective evaluations of joint disease to examine whether RA truly does improve during pregnancy and to what extent. Specifically, we aimed to answer the following questions: (1) does RA disease activity improve during pregnancy; and (2) how common is a postpartum flare in patients with RA?

\section{MATERIALS AND METHODS}

Publication search and selection of studies. This review was conducted according to guidelines of Preferred Reporting Items for Systematic reviews and Meta-Analyses (PRISMA). A systematic review of PubMed, EMBASE/Medline, Cochrane, and LactMed databases was performed (Supplementary Figure 1, available from the authors on request). Additional studies were identified through checking the reference lists and other author publications of articles selected for full-text analysis. Two independent reviewers (HJ and SL) screened the retrieved articles by reading the title and abstract to identify studies that met these inclusion criteria: human observational prospective studies, more than 5 patients per study, and containing data on RA disease activity gathered using an objective scoring system conducted by a clinician/health professional, with serial assessments of disease activity during pregnancy. Postpartum data were recorded when listed. Efforts were made to ensure duplicate data were not included, notably multiple studies derived from the Pregnancy-Induced Amelioration of Rheumatoid Arthritis (PARA) cohort study, for which only the first study was included to avoid replication of data (de Man, et al, 2008 ${ }^{3}$ ). Reviews containing no original data, conference abstracts, and non-English-language studies were excluded. Where necessary, original authors were contacted for further information.

Data collection process. A data extraction sheet was developed and its relia-

Personal non-commercial use only. The Journal of Rheumatology Copyright $\odot$ 2019. All rights reserved. 


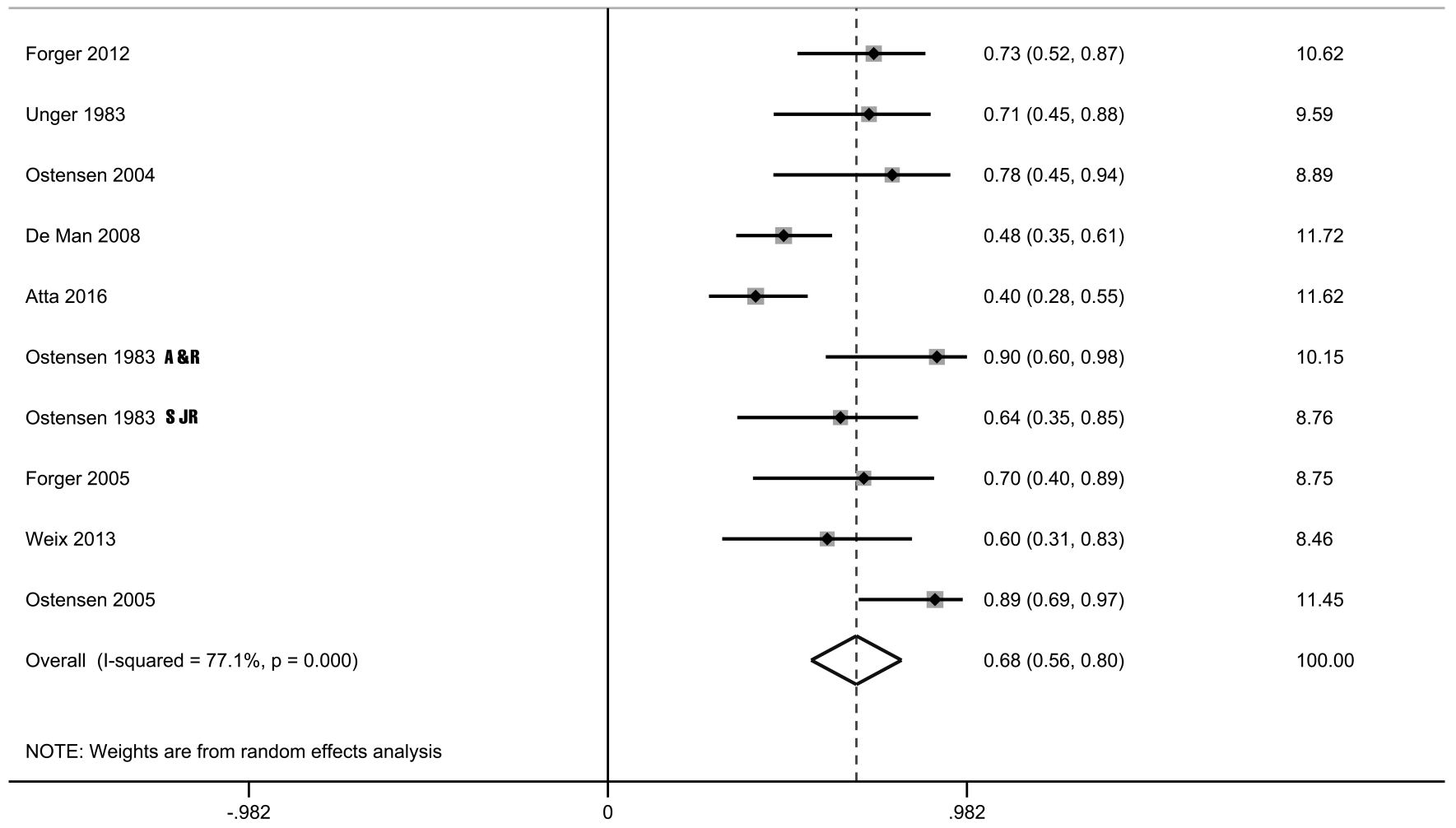

Figure 1. Forest plot of studies reporting the percentage of women experiencing improvement in rheumatoid arthritis disease activity during pregnancy. Heterogeneity between studies ( $\left.\mathrm{I}^{2}=77.1 \% ; \mathrm{p}<0.001\right)$ was noted. A\&R: Arthritis and Rheumatology; SJR: Scandinavian Journal of Rheumatology.

bility examined on 10 randomly selected studies. It was then refined accordingly to ensure that all relevant data were recorded. Two authors (HJ and SL) extracted and independently checked the data. Disagreements were resolved by discussion with author (IG). Each selected article was systematically examined to note study characteristics pertaining to the population, intervention, comparison, outcome, and time frame.

Statistics. A chi-square test for heterogeneity was performed to determine whether findings were consistent between studies. In all studies, the principal summary measure extracted from each study was the percentage of patients who showed disease improvement or deterioration noted by an improvement or decline in their disease activity scores from baseline in pregnancy or postpartum. This measure was used because exact quantification of the extent of improvement or deterioration was not possible owing to the different measures of disease activity used.

\section{RESULTS}

A total of 4673 articles were identified. Duplicate papers and those unsuitable by title alone were excluded, leaving 193 articles. Abstracts of these articles were analyzed further and from 63 articles selected for full-length review, a total of 10 studies were eligible for final analysis. Details of the number and reasons for article exclusion are listed in Supplementary Figure 2 (available from the authors on request). A total of 237 patients/pregnancies were identified from these 10 studies; prepartum data were available for 204 patients, and postpartum data were available for 135 patients. Six studies included preconception disease activity data (Ostensen 1983 A \& $\mathrm{R}^{4}$, Unger $1983^{5}$, Ostensen $2004^{6}$, de Man $2008^{3}$, Forger $2012^{7}$, and Atta $2016^{8}$ ). Five studies documented serial measurements of disease activity in each trimester and up to 3 timepoints up to 24 or 26 weeks postpartum (Ostensen $2004^{6}$, Forger $2005^{9}$, de Man $2008^{3}$, Forger $2012^{7}$, and Weix $2013^{10}$ ); 1 study compared disease activity only in trimesters 1 and 3 (Atta $2016^{8}$ ).

These 10 studies used the following disease activity scoring systems: 28-joint count Disease Activity Score (DAS28) using C-reactive protein (4 studies) $)^{3,7,8,10}$, RA Disease Activity Index (3 studies) $)^{6,9,11}$, Camp index (1 study $)^{5}$, and other scoring systems that included objective measurements, such as joint counts and grip strength (2 studies $)^{4,12}$. Heterogeneity between studies was noted $\left(\mathrm{I}^{2}=77.1 \% ; \mathrm{p}<0.001\right)$, so a random effects metaanalysis was used to obtain a pooled estimate and $95 \% \mathrm{CI}$ of the percentage with an improvement in activity score, accounting for this observed heterogeneity (Figure 1). Of the 204 pregnancies, disease activity improved in $123(60.3 \%$ overall); across studies, this varied widely from $40.4 \%$ (Atta $2016)^{8}$ to $90 \%$ (Ostensen 1983 A \& R ${ }^{4}$; Figure 2A). 


\section{\% Improved during pregnancy}

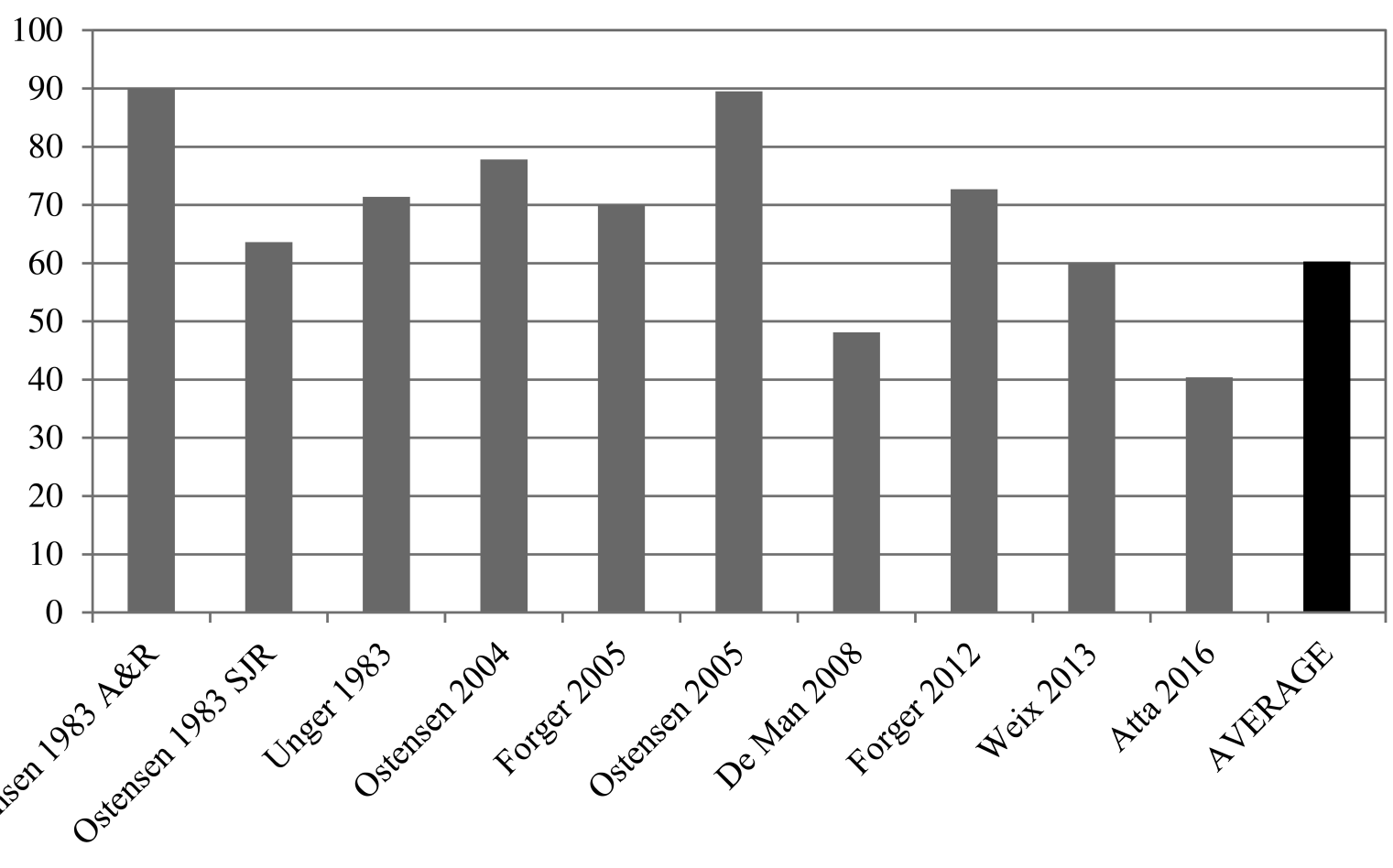

\section{\% Flared postpartum}

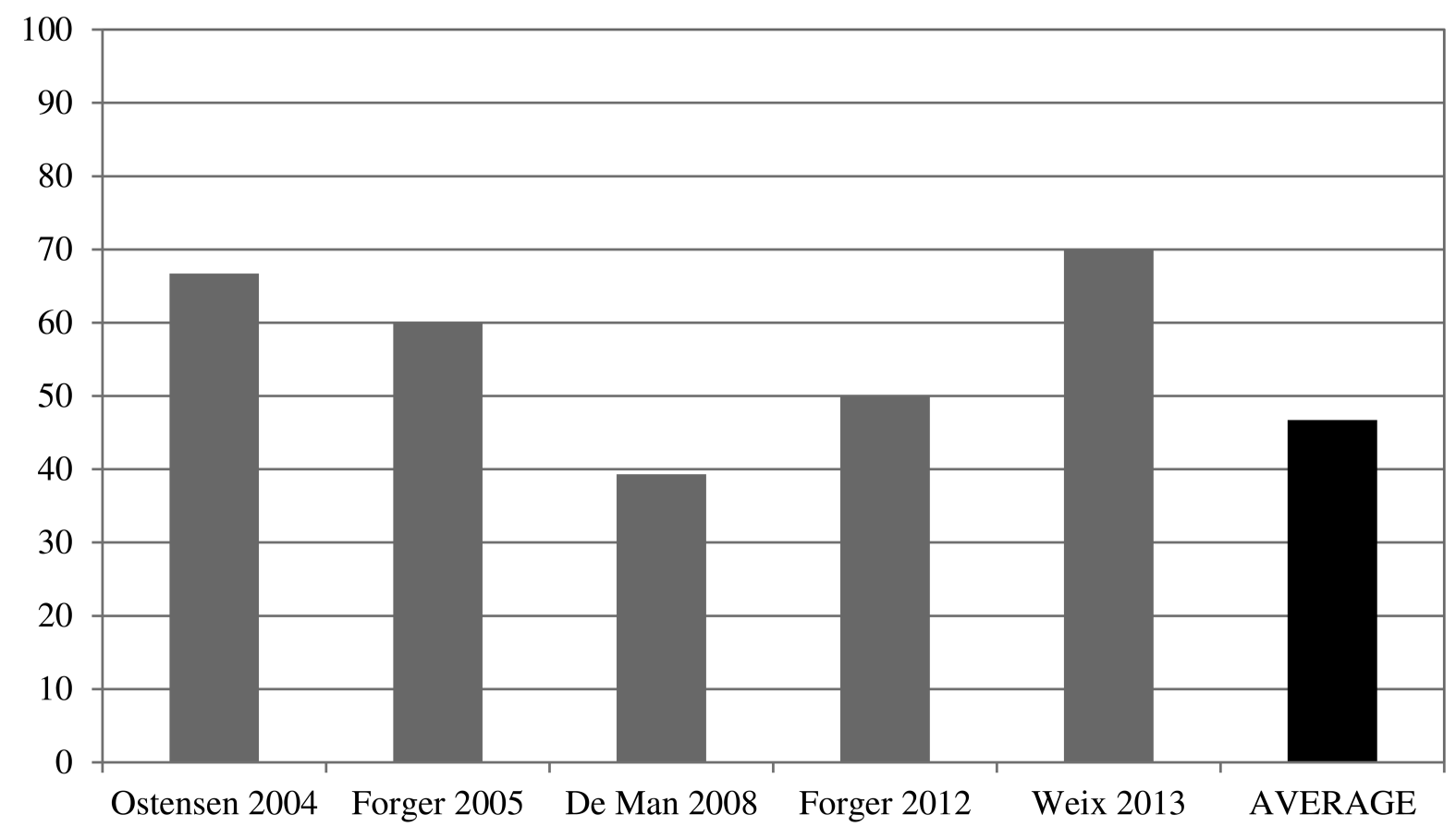

Figure 2. Percentage of patients whose disease activity improved during pregnancy (A) and relapsed postpartum (B) in each of the papers included in the metaanalysis, including overall average percentages. A. Of the 204 pregnancies for which data were available during pregnancy, percentage of improvement in disease activity scores during pregnancy varied widely, from $40.4 \%$ to $90 \%$. B. Of the 135 pregnancies for which postpartum data were available, a flare in disease activity was noted in an average of 63 patients (46.7\%). A\&R: Arthritis and Rheumatology; SJR: Scandinavian Journal of Rheumatology.

\section{Personal non-commercial use only. The Journal of Rheumatology Copyright $@$ 2019. All rights reserved.}


Data on postpartum disease activity were available in 5 papers that used a variety of disease activity scoring systems on 135 pregnancies (Table 1). All these studies included disease activity measures at 6 weeks postpartum; some also included data at 12 and 24 or 26 weeks. A postpartum increase in disease activity was noted in $63(46.7 \%)$ of these pregnancies (Figure 2B). Pregnancy outcomes were reported in only 3 studies with no increase in adverse events, although of the 135 pregnancies, 17 babies (23\%) were born prematurely and 16 babies (22\%) were small for their gestational age ${ }^{4,6,8}$.

\section{DISCUSSION}

Disease management during pregnancy is complicated by several factors including an increased burden of pregnancy morbidity and adverse pregnancy outcomes that has been linked with increased disease activity ${ }^{13,14}$. Accurate knowledge of the effects of pregnancy upon RA is therefore required for prepregnancy counseling.
Historical reports of up to $90 \%$ of patients with RA improving in pregnancy were mostly from retrospective studies, which lacked objective measures of disease activity. Findings from our systematic review of prospective studies using serial, objective measures of disease activity have revealed more modest ameliorative effects of pregnancy on RA disease activity, with only $60 \%$ of patients demonstrating improvement in disease activity. Our data also demonstrated a postpartum increase in disease activity in about half of all pregnancies.

It is important to note that the most accurate record of changes of disease activity around pregnancy are obtained when a prepregnancy assessment within a defined time frame before conception has been made and serial measurements performed in each trimester of pregnancy. Further, postpartum disease flares are best collected by at least 2 assessments within 3-6 months after delivery, when relapses of disease activity occur most frequently. This gold standard

Table 1. Summary of disease activity scoring systems used in the 10 papers evaluated for final analysis, and an indication of the different objective disease activity scoring systems and criteria for disease activity alterations used during pregnancy and postpartum.

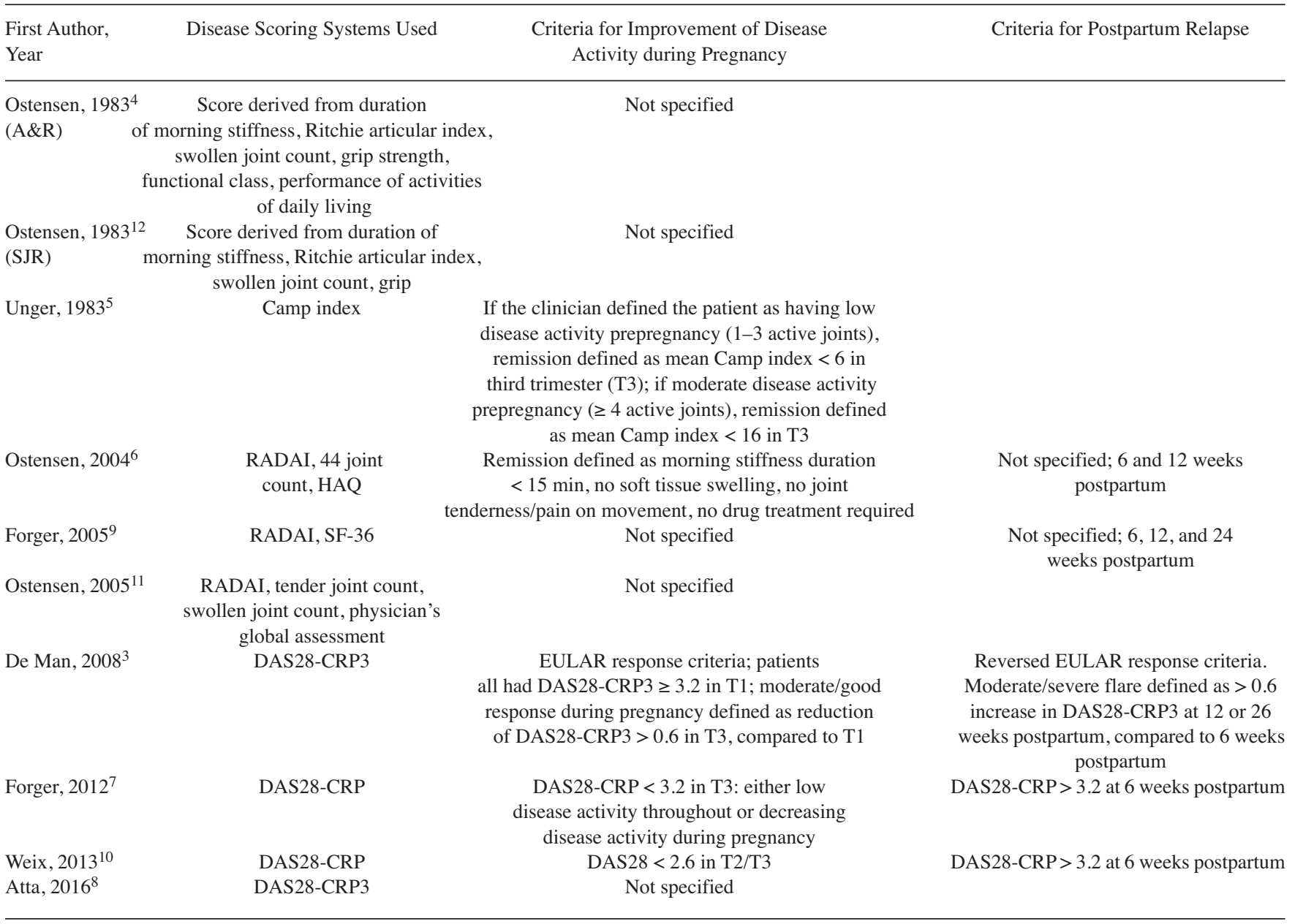

RADAI: Rheumatoid Arthritis Disease Activity Index; SF-36: Medical Outcomes Study Short Form-36; HAQ: Health Assessment Questionnaire; DAS28-CRP3: 28-joint count Disease Activity Score using C-reactive protein 3; EULAR: European League Against Rheumatism; A\&R: Arthritis and Rheumatology; SJR: Scandinavian Journal of Rheumatology. 
was not achieved in all of our selected studies, which recorded disease activity in 6 studies prepregnancy, 9 studies in all trimesters, 1 study in the first and third trimesters, 4 studies at 3 timepoints up to 24 or 26 weeks postpartum, and 1 study at only 6 weeks postpartum. Therefore, although it is conceivable that some alterations in disease activity may have been missed, we do not believe that has adversely affected our findings.

The first study by de Man, et al (2008) ${ }^{3}$ to use the DAS28 as a validated disease activity score to measure disease activity before and at multiple timepoints during and after pregnancy in the PARA cohort identified that the ameliorative effects of pregnancy on disease activity in the third trimester were more marked in those who had moderate to high disease activity in the first trimester compared to those with baseline low disease activity. Further, 39\% of these patients had at least a moderate flare postpartum. The PARA cohort has subsequently expanded and been intensely studied in further publications that include serial measures of disease activity throughout pregnancy but have focused on other aspects of RA pregnancy, such as the effect of ACPA positivity ${ }^{15}$ and influence of maternal cytokines on fetal growth ${ }^{16}$. Therefore, we included only the original PARA study in our analysis to avoid duplication of the original cohort data and thus dilution of other study findings.

Although many of the studies included data on antibody status, data were not expressed in a format by which we could interpret whether antibody status affects disease activity during pregnancy or postpartum. Forger, et al (2012), however, identified that the group of patients who entered pregnancy and continued to have high disease activity had higher levels of anticyclic citrullinated peptide antibody (anti-CCP) compared to those with low disease activity ${ }^{7}$. Subanalyses by de Man, et al (2008), however, found that presence of anti-CCP or rheumatoid factor did not alter the course of disease activity during pregnancy or postpartum ${ }^{3}$. Further, although many of the papers include some limited data on medication use, we were unable to meaningfully interpret whether this affected disease activity.

It is difficult to obtain conclusions from the papers regarding whether the patients who improved during pregnancy were more likely to flare postpartum; however, Forger, et al (2012) report that at 6 weeks postpartum, a DAS28 > 3.2 was seen in all patients who demonstrated persistent active disease during pregnancy, but in only $31.3 \%$ in those with low or inactive disease during pregnancy ${ }^{7}$.

There are several limitations to this metaanalysis, which is highlighted by the high degree of heterogeneity between studies that is likely due to differences in study design or variations in the patient population (for example, disease duration, disease severity, demographic differences). The most notable difference between study designs is in disease activity measurements and definitions of disease improvement or relapse. The papers included used different disease activity scoring systems, and even papers that used the same scoring system had different definitions of active disease to compare whether activity improved or worsened. Further, the studies were inconsistent in their methodologies, and assessed disease activity at different points during pregnancy and postpartum; therefore it is difficult to accurately compare these results. For example, the time span of assessment postpartum varied from 6 weeks to 6 months. Although treatment data were not included in our study, we note the wide time span from the earliest studies to the most current ( $>30 \mathrm{yrs}$ ), during which period there have been many alterations in treatment with the introduction of combination therapies, new drugs (particularly biologics), and treat-to-target regimens. Interestingly, studies dating from 1983 to 2005 noted a greater improvement in disease activity scores during pregnancy compared to those from 2008 to 2016 (77\% and 55.3\%, respectively). This reduction may be a consequence of these more effective treatment regimens inducing remission or low disease activity in more patients with RA, who thus plan pregnancy and experience less impressive gestational improvement.

Additionally, although all studies included either a validated disease activity score or an objective score based on clinical assessment as a primary inclusion criterion, some of them also used subjective measures. In these cases, measures of disease improvement or deterioration used for final analysis may have included subjective measures for reasons beyond our control. But upon review of the final results of papers that included some subjective measurements, we concluded that those results did not have a significant effect on our final analysis.

Our systematic analysis of prospective studies using objective markers of disease activity and related scoring systems found that $60 \%$ of patients with RA improve during pregnancy and $47 \%$ relapse postpartum. This information is important when counseling patients with RA prepartum.

\section{REFERENCES}

1. Straub RH, Buttgereit F, Cutolo M. Benefit of pregnancy in inflammatory arthritis. Ann Rheum Dis 2005;64:801-3.

2. Hazes JM, Coulie PG, Geenen V, Vermeire S, Carbonnel F, Louis E, et al. Rheumatoid arthritis and pregnancy: evolution of disease activity and pathophysiological considerations for drug use. Rheumatology 2011;50:1955-68.

3. de Man YA, Dolhain RJ, van de Geijn FE, Willemsen SP, Hazes JM. Disease activity of rheumatoid arthritis during pregnancy: results from a nationwide prospective study. Arthritis Rheum 2008;59:1241-8.

4. Ostensen M, Husby G. A prospective clinical study of the effect of pregnancy on rheumatoid arthritis and ankylosing spondylitis. Arthritis Rheum 1983;26:1155-9.

5. Unger A, Kay A, Griffin AJ, Panayi GS. Disease activity and pregnancy associated alpha 2-glycoprotein in rheumatoid arthritis during pregnancy. Br Med J 1983;286:750-2.

6. Ostensen M, Fuhrer L, Mathieu R, Seitz M, Villiger PM. A prospective study of pregnant patients with rheumatoid arthritis and ankylosing spondylitis using validated clinical instruments. Ann Rheum Dis 2004;63:1212-7.

Personal non-commercial use only. The Journal of Rheumatology Copyright $\subset$ 2019. All rights reserved. 
7. Forger F, Vallbracht I, Helmke K, Villiger PM, Ostensen M. Pregnancy mediated improvement of rheumatoid arthritis. Swiss Med Wkly 2012;142:w13644.

8. Atta DS, Girbash EF, Tharwat I, Abdelwahab SM, Abdeldayem HM, Ghonaim R. Maternal cytokines and disease severity influence pregnancy outcomes in women with rheumatoid arthritis. J Matern Fetal Neonatal Med 2016;29:3358-63.

9. Forger F, Ostensen M, Schumacher A, Villiger PM. Impact of pregnancy on health related quality of life evaluated prospectively in pregnant women with rheumatic diseases by the SF-36 health survey. Ann Rheum Dis 2005;64:1494-9.

10. Weix J, Haupl T, Raio L, Villiger PM, Forger F. The physiologic increase in expression of some type I IFN-inducible genes during pregnancy is not associated with improved disease activity in pregnant patients with rheumatoid arthritis. Transl Res 2013;161:505-12.

11. Ostensen M, Forger F, Nelson JL, Schuhmacher A, Hebisch G, Villiger PM. Pregnancy in patients with rheumatic disease: anti-inflammatory cytokines increase in pregnancy and decrease post-partum. Ann Rheum Dis 2005;64:839-44.
12. Ostensen M, Van Schoultz B, Husby G. Comparison between serum alpha 2-pregnancy-associated globulin and activity of rheumatoid arthritis and ankylosing spondylitis during pregnancy. Scand J Rheumatol 1983;12:315-8.

13. Chakravarty EF, Nelson L, Krishnan E. Obstetric hospitalizations in the United States for women with systemic lupus erythematosus and rheumatoid arthritis. Arthritis Rheum 2006;54:899-907.

14. de Man YA, Hazes JM, van der Heide H, Willemsen SP, de Groot CJ, Steegers EA, et al. Association of higher rheumatoid arthritis disease activity during pregnancy with lower birth weight: results of a national prospective study. Arthritis Rheum 2009;60:3196-206.

15. de Man YA, Bakker-Jonges LE, Goorbergh CM, Tillemans SP, Hooijkaas H, Hazes JM, et al. Women with rheumatoid arthritis negative for anti-cyclic citrullinated peptide and rheumatoid factor are more likely to improve during pregnancy, whereas in autoantibody-positive women autoantibody levels are not influenced by pregnancy. Ann Rheum Dis 2010;69:420-3.

16. de Steenwinkel FD, Hokken-Koelega AC, de Man YA, de Rijke YB, de Ridder MA, Hazes JM, et al. Circulating maternal cytokines influence fetal growth in pregnant women with rheumatoid arthritis. Ann Rheum Dis 2013;72:1995-2001. 